\title{
GOBERNANZA INTERCULTURAL Y CIUDADANÍA POLÍTICA EN PÁTZCUARO, MICHOACÁN, MÉXICO
}

\author{
Miguel Rodrigo González Ibarra ${ }^{1}$
}

\section{RESUMEN}

El tema de ciudadanía advierte un análisis desde diversas interpretaciones teóricas y metodológicas que apuntan hacia una perspectiva pluridimensional y connotaciones complejas. No obstante, existe un cierto consenso respecto a que el término ciudadanía remite a la pertenencia de los individuos y los grupos sociales a una determinada comunidad política. Este trabajo se propone analizar: a) cuál es la importancia de la ciudadanía y la gobernanza intercultural en los procesos de gobierno y construcción de políticas públicas locales; b) explicar de qué forma se concibe la construcción de ciudadanía activa a través de la llamada participación solidaria; y c) analizar cuáles fueron las aportaciones del Consejo Ciudadano de la Región Lacustre de Pátzcuaro en el Estado de Michoacán, México, hacia la construcción de ciudadanía política y gobernanza intercultural. Utilizando una perspectiva cualitativa -a través de la revisión de documentos, entrevistas en actores y análisis empírico- se discute que, en mayor o menor medida, la creación del Consejo motivó la gobernanza intercultural que requieren las comunidades para incidir en los procesos de participación solidaria y construyó las bases para una ciudadanía activa y solidaria hacia temas relacionados con la educación y el medio ambiente, entre otros temas, comunitarios.

Palabras claves: Gobernanza Intercultural. Ciudadanía. Michoacán. Consejo Ciudadano de la Región Lacustre.

\section{GOVERNANÇA INTERCULTURAL E CIDADANIA POLÍTICA EM PÁTZCUBARO, MICHOACÁN, MÉXICO}

\section{RESUMO}

A questão da cidadania adverte para uma análise de várias interpretações teóricas e metodológicas que apontam para uma perspectiva multidimensional e conotações complexas. No entanto, há algum consenso de que o termo cidadania se refere à adesão de indivíduos e grupos sociais a uma dada comunidade política. Este trabalho tem como objetivo analisar: (a) qual é a importância da cidadania e da governança intercultural nos processos de governança e construção de políticas públicas locais; b) explicar como a construção da cidadania ativa é concebida através da chamada participação solidária; e c) analisar quais foram as contribuições do Conselho cidadão da região de lacustre de Pátzcubaro, no

\footnotetext{
1 Doctor en Ciencias Políticas y Sociales por la Universidad Nacional Autónoma de México. Profesor-Investigador Titular en el Departamento de Sociología de la Universidad Autónoma Metropolitana-Iztapalapa. Orcid Id: https://orcid.org/0000-0003-3999-3532. E-mail: ibarra000@yahoo.com
} 
estado de Michoacán, México, rumo aos centros estéticos da cidadania política e do governo intercultural. Utilizando uma perspectiva qualitativa-através da revisão de documentos, entrevistas com atores e análise empírica-argumenta que, na menor medida, a criação do Conselho motivou a governança intercultural que as comunidades necessitam para Concentrar-se nos processos de participação solidária e nos fundamentos de uma cidadania ativa e solidária para questões de educação e meio ambiente, entre outros temas, a Comunidade.

Palavras-chave: Governança intercultural. Cidadania. Michoacán. Conselho de cidadãos da região do lago.

\section{INTERCULTURAL GOVERNANCE AND POLITICAL CITIZENSHIP IN PÁTZCUARO, MICHOACÁN, MEXICO}

\section{SUMMARY}

The issue of citizenship warns an analysis from various theoretical and methodological interpretations that point to a multidimensional perspective and complex connotations. However, there is a certain consensus that the term citizenship refers to the belonging of individuals and social groups to a political community. This paper intends to analyze: a) what is the importance of citizenship and intercultural governance in the processes of government and construction of local public policies; b) explain how the construction of active citizenship is conceived through the so-called solidarity participation; and c) analyze what were the contributions of the Citizen Council of the Lacustrine Region of Pátzcuaro in the State of Michoacán, Mexico, towards the construction of political citizenship and intercultural governance. Using a qualitative perspective - through document review, stakeholder interviews and empirical analysis - it is argued that, to a greater or lesser extent, the creation of the Council motivated the intercultural governance that communities require to influence solidarity participation processes and built the foundations for active and supportive citizenship towards issues related to education and the environment, among other issues, community.

Keywords: Intercultural Governance. Citizenship. Michoacán. Citizen Council of the Lacustrine Region.

\section{PRESENTACIÓN}

En los últimos años el tema de ciudadanía advierte un análisis desde diversas interpretaciones teóricas y metodológicas que apuntan hacia una perspectiva pluridimensional y connotaciones complejas. No obstante, existe un cierto consenso respecto a que el término ciudadanía remite a la pertenencia de los individuos y los grupos sociales a una determinada comunidad política. Por ciudadanía también se ha invocado a una condición natural o vecino de una ciudad y a la de habitante en de una 
ciudad como sujeto de derechos políticos y un cierto estatus legal. Sea como fuere, la noción plantea un desafío para comprender una condición relacionada con el carácter activo y participativo en los procesos de gobierno a través de la llamada gobernanza intercultural y apuntan a la influencia que se tiene por parte de grupos, organizaciones e individuos en la construcción de políticas públicas, especialmente en el ámbito de gobierno local.

El objetivo de este trabajo es: a) analizar cuál es la importancia de la ciudadanía y la gobernanza intercultural en los procesos de gobierno y construcción de políticas pública locales; y, b) explicar de qué forma se concibe la ciudadanía activa a través de la llamada participación solidaria generada por el Consejo Ciudadano de la Región Lacustre de Pátzcuaro (Consejo en adelante) en el Estado de Michoacán, México. Como hipótesis, se plantea que, en mayor o menor medida, el Consejo surgió en base al carácter activo y solidario de sus fundadores para generar acción colectiva y movilización hacia el espacio público y con ello motivar la gobernanza intercultural que requieren las comunidades para incidir en los procesos de participación en esta región del país; la creación del Consejo se propuso la construcción de ciudadanía activa para influir en el diseño de una agenda ciudadana relacionada con la cultura, la educación y la defensa del medio ambiente, entre otros (formales e informales) al margen del gobierno y los partidos políticos desde una perspectiva de bien común, filantrópica y ciertamente voluntaria para motivar el cambio social.

La exposición de este trabajo se articula de la siguiente manera: por un lado, se realiza una discusión teórica respecto a la noción de gobernanza intercultural y se plantea un análisis sobre la noción de participación solidaria para contextualizar el surgimiento del Consejo; por otra parte, se presenta una síntesis - a través del enfoque de investigación cualitativa con base en entrevistas realizadas en actores claves y sistematización de documentos- sobre la gestación y las acciones emprendidas por esta organización. Finalmente, se presenta una reflexión crítica sobre la restauración del edificio de una biblioteca y la rehabilitación del rio Guaní 
que atraviesa la ciudad de Pátzcuaro y que, en su conjunto, consideramos son evidencias acerca de la construcción de redes sociales e institucionales para impulsar el bien común a través de la construcción de ciudadanía, participación solidaria y la gobernanza intercultural en el occidente de México.

\section{GOBERNANZA INTERCULTURAL}

En los últimos veinte años llama la atención en la mayor parte de los países latinoamericanos, especialmente en México, analizar y repensar los fundamentos del paradigma democrático $y$, según diversos informes de organismos internacionales (PNUD, 2014), un número importante de personas en situación de privación está dispuesta a dejar la democracia a cambio de un mejor bienestar material. En este sentido, y desde la perspectiva de Aguilar (2007), existe un discurso acerca de los procesos de gobierno que refiere a que la crisis de los Estados sociales se manifiesta en la disminución de las capacidades estatales para atender con éxito la creciente demanda de bienes, recursos y servicios sociales. La crisis tiene un efecto en la atención de los problemas públicos, integración social y pérdida de la legitimidad de las instituciones políticas, así como en la ruptura de los procesos de cooperación y solidaridad grupal que afecta la deliberación y acción colectiva. En este proceso, y frente a la exigencia de una gestión pública más eficaz y eficiente, la promoción de la participación social y política -por medio de la gobernanza y los procesos de incidencia pública de organizaciones de la sociedad civil- se hacen indispensables para reconstituir nuevas formas de innovación democrática y revaloración del espacio público.

Si bien las políticas públicas articuladas desde un sentido horizontal proponen dar nuevas pautas de coordinación a la acción colectiva, el concepto de gobernanza ha venido a motivar el papel de las organizaciones autónomas en los procesos de definición de la política y las políticas. Sahuí (2009), señala que para generar bienestar, justicia e igualdad es necesario considerar un reposicionamiento de los agentes sociales en el 
proceso de gobierno. En efecto, la gobernanza implica una nueva correlación de fuerzas de los agentes, individuales y colectivos, respecto a la administración burocrática centralizada como única encargada de la gestión pública. El discurso de la gobernanza es a favor de abrir y democratizar al gobierno a través de la inclusión de voces y representaciones de los ciudadanos en la deliberación colectiva y para generar agenda política.

Desde otra perspectiva, a la concepción normativa de la democracia se le invita revalorar los objetivos de justicia o equidad que involucre un aspecto instrumental en el proceso de desarrollo de un país. La gobernanza plantea la inclusión de todos los actores en la toma de decisiones colectivas y una apertura a todas las personas en los procesos de deliberación y representación más allá de su condición o capacidades. En este sentido, se advierte la necesidad de generar un diseño institucional que permita lograr una inclusión real y evitar la "captura" por parte de las elites o grupos de todos los beneficios del proceso político. Así, es posible la existencia de una relación entre democracia deliberativa y gobernanza que motive y potencie la participación ciudadana en los asuntos públicos. La democracia deliberativa implica una comprensión acerca del interés común, la acción colectiva y el diálogo entre los actores bajo un principio de inclusión democrática.

Pese a que existe una literatura amplia y enfoques diversos sobre la noción de gobernanza, se puede inferir la necesidad de un construir un estilo de gobierno caracterizado no solamente por un mayor grado de cooperación e interacción entre el Estado y los actores no estatales en el interior de una red decisionales mixtas entre lo público y lo privado, sino también es posible señalar "la creación de una estructura que es resultado del interés público y procesos mediante los cuales se llevan a cabo prácticas de intercambio, coordinación, control y adopción de decisiones en los sistemas democráticos (NATERA, 2005: 759)". En este marco, se infiere que la gobernanza tiene un común denominador que radica en que el Estado ha dejado de tener el monopolio sobre los conocimientos y sobre los recursos 
económicos e institucionales necesarios para gobernar, así como supone la influencia de los actores no gubernamentales en el proceso político debido a, entre otros factores, la crisis fiscal del Estado y la necesidad de generar una mayor eficiencia en la producción de servicios públicos e incluso replantear las funciones gubernamentales hacia otra forma de gobernar y encauzar la planificación democrática ante el cambio, la complejidad y la diversidad que enfrentan los gobiernos.

En la actualidad cuando se dice que es necesario generar un estilo de gobernar diferente a las formas tradicionales y verticales de gobierno, se alude a la renovación de capacidades del Estado, y del propio gobierno y la administración pública, para enfrentan la diversidad de problemas públicos y acción colectiva que se producen en diferentes esferas entre lo público y lo privado. La transformación de las estructuras no es un proceso aislado de los actores sociales, sino implica un trabajo conjunto para fortalecer el diseño institucional y dar cabida a las demandas y expresiones de la sociedad con su entorno. Leroux (2005), desde esta posición, advierte que la gobernanza pone como centro de atención la identificación de pautas de conducta, procesos operativos y estilos de gobierno que requieren de mejores métodos y mecanismos para impulsar una gestión pública, que se complementen y entiendan para encauzar el cambio social.

En otro orden de ideas, la gobernanza se refiere a un cambio en el equilibrio entre el Estado y la sociedad, y centra su propuesta en la incidencia política que tienen los individuos, grupos, y organizaciones en la construcción de políticas públicas y en el diseño institucional que permita no sólo generar espacios de mayor deliberación sobre asuntos públicos, sino definir mecanismos de mejora a la gestión pública democrática. De acuerdo con Vargas (2016: 5), la creación de sistemas de gobernanza intercultural se sustenta en políticas públicas y programas democráticos desde una visión de diversidad cultural para la promoción del desarrollo (económico, social y político) y con la finalidad de lograr la paz mundial.

En este sentido, se entiende por gobernanza intercultural "a las demandas relacionadas con la gestión de la diversidad que interpela a la 
gestión del gobierno local da dar una respuesta (VARGAS, 2016: 7)." La gobernanza intercultural implica la capacidad de gestión de las políticas interculturales a través de dispositivos y mecanismos de operación necesarios para desarrollar políticas en todas las funciones administrativas y actividades de dirección y coordinación de los gobiernos locales. En este proceso la gobernanza intercultural establece los objetivos para encauzar el ejercicio de gobierno, así como propone el fortalecimiento de las capacidades institucionales para analizar la diversidad cultural y proponer mejores diseños institucionales, así como una mejor distribución de recursos entre los grupos y organizaciones de la sociedad civil. La gobernanza intercultural aporta, en esta línea, al trabajo de innovación de la gestión pública al considerar una perspectiva más abierta a los temas, preocupaciones y casos de éxito en que la participación política de los ciudadanos produce un impacto en el desarrollo de la comunidad. En síntesis

Vargas (2016), aporta al debate que la gobernanza intercultural juega un papel relevante en la diversidad y sus implicaciones en lo político, económico y social. La interculturalidad se sustenta no sólo en valores como la confianza, la transparencia y la rendición de cuentas, sino constituyen el eje articulador de las interacciones entre los ciudadanos y las instituciones. En este ejercicio el concepto advierte la creación de políticas y estrategias para el desarrollo social, formación de liderazgos, fortalecimiento de programas sociales y mecanismos para canalizar la opinión, diversidad y propuestas de las personas y sus comunidades. Además, este ejercicio convoca a potenciar los derechos humanos y sociales, así como motiva la construcción e incidencia política de grupos y nuevas identidades emergentes para gobernanza democrática. En síntesis: "El reconocimiento de la gobernanza intercultural alienta los derechos humanos universalmente reconocidos y garantizados para cada persona en su pleno ejercicio, lo que a su vez refuerza la cohesión social y la renovación de modos y formas de gobernanza democrática (VARGAS, 2016:10)." 


\section{CIUDADANÍA SOLIDARIA Y FILANTROPÍA COMUNITARIA}

La participación ciudadana se sustenta en la articulación de procesos sociales que implica la agrupación de individuos en organizaciones, grupos y colectivos de la sociedad civil para promover la defensa o representación de sus intereses políticos (CUNNILL, 1991, CANTO, 2010, GONZÁLEZ, 2011; GONZÁLEZ, 2005). Precisamente, el desarrollo de la vida política y democrática de una comunidad implica que los ciudadanos desarrollen en su práctica social procesos de interacción con la finalidad de favorecer el tejido social e innovar formas de participación social e intervención en lo público. En esta línea, la participación alude al desarrollo comunitario a través de un conjunto de acciones desarrolladas por diversos sectores y que buscan una solución a sus necesidades y demandas específicas en diversos espacios y ámbitos de la sociedad civil.

Un tipo de participación que en los últimos años ha generado innovación democrática en el análisis de los procesos de buen gobierno y construcción de ciudadanía, es la participación solidaria que se distingue por generar acciones voluntarias por diversos sectores de la comunidad y que tienen como finalidad coadyuvar a la búsqueda del bien común y el establecimiento de proyectos autónomos para atender necesidades específicas y complementar -en ciertos casos- acciones que el Estado y las instituciones han dejado de atender o no existen los impactos y métodos adecuados para su desarrollo en la comunidad.

Butcher, (2008: 37) ha señalado que una acción voluntaria en la comunidad puede ser entendida como la labor que realiza una persona por elección propia y sin recibir remuneración aporta tiempo a una actividad que va más allá del ámbito familiar en el servicio de los demás para el beneficio de terceros de la sociedad en su conjunto. En este sentido, la acción voluntaria implica una praxis que se convierte en motor para el engranaje social y esta fuerza permite coadyuvar en el desarrollo de una comunidad con lazos de confianza y donde se promueve la cooperación, la participación en los asuntos públicos y existe cierta consciencia para la mejora comunitaria, inclusión social e iniciativas hacia la agenda pública. 
Por comunidad entendemos a un espacio físico y social en que se despliegan y entrelazan actividades de gestión, organización y participación solidaria (ANDER-EGG, 2003). La comunidad es un área de intervención para lograr los propósitos del desarrollo a partir de políticas y proyectos concretos. De esta manera, la participación solidaria advierte actividades e iniciativas conjuntas individuales y grupales para el logro de objetivos en beneficio del colectivo y de la comunidad política a la que se pertenece.

La comunidad es también un espacio micro-social donde la cooperación, intercambio y ayuda mutua, aportan a la creación de los procedimientos de gestión, programas y proyectos hasta alcanzar el nivel macro-social. La comunidad es un ámbito de convivencia, aprendizaje y coordinación para el desarrollo donde las aportaciones locales son la base para la construcción de alternativas para el desarrollo comunitario y la llamada filantropía comunitaria entendida como aquellas acciones que se llevan a cabo por sujetos a través de acciones o participación solidaria por amor al ser humano.

En este orden de ideas, y pese a que el concepto de filantropía (REYGARCÍA, 2013, 7) es muy remoto y existe un debate importante sobre la labor que realizan organizaciones de la sociedad civil, la filantropía comunitaria puede ser concebida como la actividad solidaria que lleva a cabo un conjunto de personas de una misma localidad para dar y motivar la ayuda a sí mismos y que comparte recursos para el bien común, así como es promovida por las tradiciones, la cultura e incluso religiosas.

La European Foundation Centre, señala que la filantropía comunitaria se define como "[...] el acto de ciudadanos e instituciones locales que aportan recursos o bienes, además de su tiempo y habilidades, para promover el bienestar de los demás y el mejoramiento de la comunidad en la que viven y trabajan (KNIGHT, 2012: 3)." En esta definición se advierte que la filantropía comunitaria puede expresarse de manera formal, informal y espontánea a través de la contribución de los ciudadanos a organizaciones que se crean y se dan a la tarea de utilizar los recursos y contribuciones para impulsar proyectos que mejoren la calidad de vida de sus habitantes. 
La filantropía comunitaria se sustenta en los valores, intercambio y la reciprocidad basada en el principio de solidaridad, el cual es el elemento principal para la construcción de vínculos, confianza institucional, liderazgo comunitario, capital social y fomento a la sustentabilidad y promoción de la inclusión social y equitativa. En este sentido, se puede inferir que la filantropía comunitaria contribuye al establecimiento de nuevos temas en la construcción de ciudadanía y fomenta la participación solidaria por medio de la cooperación para el desarrollo de la comunidad.

Consideramos, que la filantropía comunitaria motiva los procesos sociales y genera entre los individuos el interés para participar en procesos de incidencia política y acción colectiva que, de manera voluntaria, formal e informal, tiene como interés resolver conflictos, participar en proyectos sociales, construir ciudadanía activa y desarrollar un marco de referencia hacia el bien común que permita involucrar a los más excluidos a través de la interacción y el trabajo solidario.

\section{CONSTRUCCIÓN DE CIUDADANÍA EN EL CONSEJO CIUDADANO LACUSTRE}

El análisis de las organizaciones de la sociedad civil y de los procesos de movilización social hacia la agenda de gobierno y la construcción de políticas públicas es posible comprenderlo por medio del concepto de acción pública, el cual permite estudiar y entender los procesos participativos en la construcción de políticas públicas (COBB Y ROSS, ROSS, 1976). Según Cabrero, la acción pública puede entenderse como "Ia construcción y la calificación de los problemas colectivos en una sociedad, problemas que ella delega o no, en una o varias autoridades, en su totalidad o en partes, para la elaboración de respuestas, de contenidos y de procesos para su tratamiento (CABRERO, 200: 2)." En efecto, el análisis de los procesos de participación solidaria y las iniciativas de filantropía comunitaria toman forma por medio del análisis específico de las organizaciones, formales e informales, que se constituyen $u$ organizan para efectos de llevar a cabo una acción colectiva y desplegar procesos de incidencia y acción pública. 
En el caso del Consejo Ciudadano de la Región Lacustre de Pátzcuaro, A.C. (en adelante se definirá como Consejo) es importante contextualizar cuáles son las razones que motivaron la acción colectiva y en qué se sustenta el trabajo de participación solidaria para el desarrollo comunitario en el municipio de Pátzcuaro y especialmente en la Cuenca del Lago del mismo nombre. Este lugar se caracteriza por tener una superficie de 1.096 kilómetros cuadrados de los cuales, un área de $100 \mathrm{~km} 2$ corresponden al lago (incluyendo islas) y los $900 \mathrm{~km} 2$ restantes al área terrestre de captación en la parte occidental del país, en el Estado de Michoacán de Ocampo (Véase https://digaohm.semar.gob.mx/cuestionarios/cnarioPatzcuaro.pdf).

Los municipios más importantes dentro de la cuenca son los que están localizados en la ribera del lago (Pátzcuaro, Erongaricuaro, Tzintzuntzan y Quiroga), ya que entre los cuatro abarcan el $80 \%$ de la superficie de la zona y donde se pueden reconocer localidades con características desiguales como el número de habitantes, forma de gobierno, aspectos sociales, entre otras, pero que comparten el mismo sistema hídrico, vías de comunicación, cultura, paisaje y otros elementos ecosistémicos. En particular, en este lugar están presentes diferentes problemas asociados con el acceso, uso, conservación y restauración de los ríos y recursos naturales en los cuales se encuentran implicados actores sociales y gubernamentales en distintos ámbitos, órdenes y responsabilidades de gobierno.

En los últimos años se han realizado estudios e investigaciones que han puesto en la agenda pública la discusión para articular una política pública integral para la restauración del ecosistema en esta región. Así, se ha detectado la presencia de grupos, organizaciones civiles e instituciones que se han movilizado para acceder al tema y aprovechar los recursos que provee la región. No obstante, y si bien es posible comprender que existe un conjunto de valores, reglas, usos y costumbres y normas, entre otros aspectos, que dan forma a las relaciones entre el Estado y la sociedad, en este lugar la organización de la gestión ambiental advierte una variedad de preferencias y conflictos sociales que abonan al deterioro del ecosistema lacustre. 
Así, es pertinente la síntesis que realiza Huerto y Vargas (2014: 182-190), acerca de que desde hace más de cuatro décadas se han impulsado varias experiencias significativas para la gestión de cuencas y la implementación de políticas públicas para frenar el deterioro ambiental. No obstante, es en la década de los ochenta cuando se intensifican las estrategias de recuperación debido al incremento del deterioro de los recursos naturales y de la presión de los habitantes para establecer acciones de gobierno. Hacia la década de los noventa se inicia un esfuerzo para reorientar la política ambiental y se suman académicos, empresarios y organizaciones para perfilar un nuevo modelo de gestión ambiental desde un enfoque descentralizado y al modo de la gobernanza ambiental.

Sin embargo, y no obstante que el Lago de Pátzcuaro ha sido objeto de una larga lista de intervenciones desde 1929, entre el año 2000 y 2005, se llevaron a cabo acciones para promover proyectos acuícolas, ordenamiento pesquero, así como recuperación y limpieza el lago, principalmente. En años recientes, una gran parte de la labor realizada por los pobladores e instituciones del lugar se ha concentrado en la recuperación ambiental con un enfoque sustentable que permita elaborar diagnósticos apegados a la realidad del lugar y definir un plan de manejo integral y con visión de largo plazo.

Vargas (2014:186) apunta que los estudios han sido importantes para avanzar en el análisis del problema, pero en ciertos momentos los pescadores no han estado de acuerdo con los términos y las metodologías en los que se llevarán a cabo y, en algunos momentos, derivaron en tensión y violencia física entre pescadores regidos por el marco institucional comunitarios y pescadores fundamentados en la racionalidad de producción comercial. En este proceso la desconfianza institucional y la crítica hacia las intervenciones realizadas por el gobierno y grupos ligados a iniciativas de partidos políticos ha sido permanente debido al uso político e intereses económicos que prevalecen en la región.

Al respecto, un estudio de Ortiz (2004, citado por Vargas 2014:187) señaló que una de las fallas de la política pesquera y de llevar a cabo la 
política pública se refiere a pensar en esquemas predefinidos que no se adaptan a las condiciones actuales, como los programas federales impulsados de forma vertical y con escasa efectividad; la confianza excesiva en los mecanismos de votación para decidir en lugar de negociar y dialogar consensos, los cambios tecnológicos detectados en el sector, las intervenciones que afectan el conocimiento tradicional, los valores comunitarios y las autoridades comunitarias, la dependencia exagerada de la ayuda internacional y exógena que no toma en cuenta a las instituciones y conocimientos autóctonos y la corrupción y otras formas prácticas clientelares y oportunistas.

A pesar de que han existido diferentes asociaciones civiles que se han preocupado por impulsar un diseño de política públicas de carácter integral y con pleno respeto a las comunidades y sus liderazgos, recientemente se formó el Consejo Ciudadano de la Región Lacustre de Pátzcuaro, Asociación Civil. Este Consejo surgió en el mes de noviembre de 2015, como resultado de la preocupación de una serie de personas notables que se agruparon en la comunidad para agruparse solidariamente y reunir esfuerzos para "hacer algo por Pátzcuaro" y participar en diferentes proyectos relacionados con el desarrollo sostenible y la gestión ambiental municipal en la cuenca del Lago de Pátzcuaro.

De acuerdo con su presidente René Ortiz Rosillo, las preocupaciones para generar acción colectiva se derivan de situaciones y problemas sociales que los gobiernos (federal y local) no han sabido atender para preservar la región lacustre y, por el contrario, han abonado al descrédito de la acción de gobierno y el desarrollo comunitario. El principal problema que motivó la fundación del Consejo fue luchar por la rehabilitación del Río Guaní y su deterioro ambiental en su curso hacia la cuenca del lago de Pátzcuaro, así como en temas relacionados con la cultura y la educación como el caso de la principal biblioteca llamada Gertrudis Bocanegra donde se ubica el Mural "Historia de Michoacán" de Juna O "Gorman, entre otros temas vinculados a la memoria histórica del lugar y edificios coloniales. 
Como una gran parte de organizaciones civiles, el Consejo inició a través de vínculos familiares y redes para impulsar el trabajo comunitario. A este respecto su fundador señala que existía una cierta experiencia en actividades de cooperación con el gobierno municipal y a través de actividades de apoyo hacia temas relacionados con la política de salud y del cual incluso fue invitado por las autoridades para intervenir en la administración pública del ayuntamiento.

"[...] yo había participado no en asociaciones civiles, había participado en un partido político y, este, fui Presidente del Comité Municipal, del Comité Distrital y fui secretario del Ayuntamiento de Pátzcuaro; en alguna ocasión la que iba a ser la candidata me pidió que si le daba una opinión de lo que había que hacer en salud del municipio y le preparé un escrito [...] y a razón de eso me interesé y luego me invitaron para participar como Secretario del Ayuntamiento en el cual duré dos años (ORTIZ, 2018)."

Como muchos procesos de constitución de organizaciones civiles, el Consejo se estructuró como parte de déficit que se presenta en estas poblaciones para impulsar la construcción de ciudadanía e interés de la población para colaborar en la discusión de los asuntos públicos y que, en menor o mayor medida, se ve afectada por la inseguridad, la violencia política y las redes de narcotráfico que prevalecen en este lugar.

En este marco, los integrantes del Consejo se interesaron en la difusión del tema y en consolidar una estructura organizativa sólida que le permitan lograr consistencia para el trabajo colectivo. En su mayor parte, sus miembros no son originarios de Pátzcuaro, pero se encuentran arraigados en el lugar; son profesionistas, jubilados o retirados de su profesión. De las 23 personas que iniciaron la asociación, en la actualidad sólo intervienen 12 en las reuniones de trabajo y forman parte de las comisiones sustantivas del Consejo. Además, se dividen tareas de una manera hasta cierto punto espontánea y consistente debido a que se dedican a otras labores. El tiempo que dedican a las tareas del Consejo no es de tiempo completo, pero se reúnen en promedio cada quince días para conversar y atender los temas que se derivan de sus proyectos principales. 
En el imaginario y los estatutos que se acordaron del Consejo se establece que el trabajo de la Presidencia del Consejo será de un periodo de tres años con carácter rotativo y donde se establecen comisiones que permiten la apertura y participación de sus miembros. Si bien no se cuenta con un domicilio definido, ni se tienen instalaciones fijas (muebles, equipo, telefonía, internet, etcétera), se cuenta con un espacio para realizar sesiones ordinarias y extraordinarias.

La participación de los miembros del Consejo es más voluntaria que obligatoria y sus socios (activos o no) aportan recursos (materiales y simbólicos) de forma solidaria para impulsar la acción colectiva. Como bien lo apunta René Ortiz, director del Consejo:

"[...] de acuerdo con los estatutos nosotros señalamos no tener
reuniones, sino sesiones ordinarias y extraordinarias, cuando fuera así
el caso, este, las llevamos en un ambiente muy sin tanto formalismo,
pero con resultados [...] la frecuencia con que se realizan las sesiones
son cada quince días en tiempos tranquilos [...] cuando tenemos
evento en puerta son cada semana [...] la votación de temas es a
mano alzada, directa [...] haya diversidad de diferencias, pero
buscamos las coincidencias, tratamos de ser lo más tolerantes
posibles [...] (ORTIZ, 2018)."

Desde la perspectiva del director del Consejo, el trabajo de esta asociación es casi de amigos, se trabaja en equipo y se realizan actas donde se anotan los temas tratados y los acuerdos tomados. Para llevar a cabo sus proyectos, la entidad trabaja y se articula en red con otras organizaciones e instituciones académicas relacionadas con el ámbito educativo y que se encuentran localizadas en el municipio de Pátzcuaro vinculadas a los temas culturales y ambientales. Asimismo, y se promueven relaciones con grupos sociales e indígenas de la región. En el caso de la relación con partidos políticos y organizaciones religiosas, se advierte una nula o escasa relación pese a que algunos de sus miembros son simpatizantes o militantes con algunos de ellos y se asevera que se mantienen al margen.

"[...] con organizaciones indígenas si tenemos relación, con Jarácuaro [...] la relación con los partidos, y como lo dicen los estatutos nada de política, pero si dentro de la asociación hay gente que es del PRl, que es del PRD, que es del PAN, pero nos respetamos, procuramos hasta ni hablar mucho de política y este cada quien ve 
aparte, la asociación es sin fines de lucro, sin fines políticos ni religiosos [...] la relación que hay con partidos políticos nos conocemos pero más bien nos acercamos a los municipios porque puede haber proyectos que tengan alguna relación o que requieran de la aprobación y el visto bueno de las autoridades municipales (ORTIZ, 2018)."

En este orden de ideas, sólo se coincide que existe un cierto trabajo de colaboración e intercambio con otras organizaciones -e incluso otro Consejo- y se comparten ideas, por ejemplo, con el Consejo Ciudadano de Pátzcuaro que promueve también el tema del lago. La participación política de los miembros del Consejos en otras actividades políticas se da a título personal y la relación con los partidos es más limitada, aunque existe la posibilidad de realizar algún foro de trabajo. Pese a que los problemas ambientales de la cuenca algunos trabajos la han definido como un asunto técnico-político, el Consejo a través de su estructura organizativa no ha promovido la movilización social a través de marchas, plantones o bloqueos. Más bien, sus estrategias de movilización se han orientada a seguir los cauces institucionales, el diálogo y la discusión colectiva para poder participar en la discusión de la agenda política ambiental y educativa.

Es importante mencionar que la participación solidaria que se promueve para las personas que desean entrar al Consejo se plantea de forma voluntaria y se espera de los interesados muchas ganas de colaborar más allá de sus intereses personales. Desde una perspectiva general, el Consejo considera a la participación solidaria como aquella labor que realizan los individuos en el proceso de gobernanza e incluye la colaboración en proyectos locales de desarrollo, representación en juntas o consejos de consulta con el gobierno local. Se puede definir como una acción voluntaria con un rasgo importante de participación cívica.

\footnotetext{
"Para ingresar a la asociación, está en los estatutos, generalmente si buscamos un perfil de personas que no vaya a ocasionar algunos conflictos, que no tenga intereses personales para partidos $u$ otra cosa, que sea neutro y que de verdad demuestre su amor por Pátzcuaro. El criterio de selección es que sea invitado por dos miembros del Consejo y que nos den una seguranza de cómo es [...] les damos los estatutos y les decimos como trabajamos, tenemos una cuota que es simbólica, lo digo yo, que son para copias, que son cien pesos cada mes [...] una vez que se analizan todos los criterios
} 
pues se decide si entra o no, hay varios casos en que comienzan a ir a las reuniones pero solitos luego no siguen, porque se dan cuenta que hay que trabajar que no es nada a cambio, que es voluntario y que hay que dar tantito (ORTIZ, 2018)."

Los vínculos e intercambios que se desarrollan para promover la participación social y el desarrollo comunitario, y como se expondrá en el siguiente punto, se lleva a cabo más a nivel del gobierno local y en temas específicos relacionados con la gestión del medio ambiente y el sector educativo. En este sentido, no está de más destacar que la construcción de políticas públicas implica la cooperación de esfuerzos para intentar incidir en la definición de la agenda política y coadyuvar en la legitimidad del sistema político, ya que la tendencia en muchos de los gobiernos locales, especialmente en el Estado de Michoacán, es la presencia de movilizaciones o protestas sociales debido a la incapacidad de generar inclusión social en las políticas públicas.

En este orden de ideas, entre los años de 2015 y 2018, el Consejo impulsó dos proyectos donde se han aprovechado las redes de intercambio con líderes sociales y comunitarios. La realización del primer foro sobre ríos y lagos ha sido uno de los logros relevantes con la presencia de veinticinco científicos de México y especialistas de otros lugares, ya que, a través de la colaboración con los expertos participantes se está planeando el proyecto de limpieza del Río Guaní. El proyecto, hasta ahora más exitoso, es la restauración de la Biblioteca Gertrudis Bocanegra y su mural "Historia de Michoacán" pintado por Juan $O$ "Gorman. Aunque pocas veces se ha promovido la difusión de los proyectos de forma masiva, se utilizan los medios electrónicos como Facebook, WhatsApp, el correo electrónico y los medios de difusión locales. En este sentido, la prospectiva que se plantea el Consejo es promover sobre todo la participación solidaria en las escuelas para atender y formar ciudadanía desde las escuelas, ya que- como lo dijo el director- hay muchos problemas que no acaban y tenemos que tener metas a corto y mediano plazo, y tener gente que sepa, como es el caso del Río Guaní. 
En opinión del médico cirujano René Ortiz construir ciudadanía significa permanecer juntos, aunque se infiere que es necesario construir capacidades de organización y acción colectiva en el mediano plazo. En este sentido, se dice que:

\begin{abstract}
"Buscamos la forma de seguir juntos, aunque seamos tres, pero lo ideal es que seamos más, gente que trabaje y ayude [...] todo mundo habla de participación ciudadana pero quizá no nos damos cuenta del valor, la capacidad y el poder que puede tener la ciudadanía [...] ahora que hemos tenido algunos eventos y temas de éxito, si se puede, quizá esa es la parte más importante de decir que si podemos organizarnos y ser el contrapeso de lo que el gobierno no ha hecho, yo creo que ahora el gobierno municipal esta así como [...] ellos tendrían la obligación de trabajar como el caso del proyecto de la biblioteca [...] (ORTIZ, 2018)."
\end{abstract}

\title{
EL CONSEJO COMO MOTOR DE LA CIUDADANÍA POLÍTICA
}

El análisis de la actividad que llevan a cabo las organizaciones de la sociedad civil en un problema público tiene como su principal objetivo lograr la transformación y crear capacidades para articular proyectos con un propósito solidario. En este sentido, los estatutos del Consejo referido establecen que "el objeto de la asociación es iniciar, promover, estimular, fomentar, patrocinar o directamente administrar y dirigir toda clase de actividades relacionadas con el rescate, la protección y la conservación del patrimonio cultural y natural en el territorio y la cuenca del lago de Pátzcuaro (ESTATUTOS, 2015)."

Con esta base el Consejo, desde su fundación en noviembre de 2015, ha considerado 25 proyectos posibles de intervención desde la impartición de cursos, seminarios y diplomados hasta la realización de toda clase de actividades permitidas por la ley, con fines no lucrativos, la protección, conservación, restauración y recuperación del patrimonio cultural del municipio, y coadyuvar en el aprovechamiento de los recursos naturales y la protección del ambiente. De este catálogo de acciones el Consejo decidió iniciar con dos proyectos: uno, sobre la restauración del edificio de la biblioteca pública Gertrudis Bocanegra y, otro, el de la rehabilitación ecológica del río Guani. A continuación, se comparte una síntesis de la 
participación solidaria y la incidencia pública que lleva a cabo el Consejo en estos dos proyectos

La restauración de la Biblioteca Pública "Gertrudis Bocanegra". Durante 39 años, una de las fundadoras del Consejo Ciudadano, Gloria Blancas López, laboró como bibliotecaria en la biblioteca pública Gertrudis Bocanegra. En este tiempo no sólo atendió las actividades bibliotecarias, sino cuidó el mural de Juan O "Gorman al interior del edificio donde exhibe "La historia de Michoacán." Desde la década de los 90 del siglo anterior, e inspirada por el mural, empezó a combinar su trabajo con la conservación de la cultura de la región. Junto con los niños y jóvenes que frecuentaban la biblioteca puso el altar con ofrendas para la noche de muertos, el nacimiento con figuras de la región para la navidad, así como los altares de dolores durante la semana santa.

\begin{abstract}
"Duré casi 39 años en la biblioteca Gertrudis Bocanegra de Pátzcuaro donde me interesé en las actividades de cultura de la región lacustre. Es un lugar histórico y convivir estos años en esta biblioteca me llevaron a compaginar con el rescate de nuestras tradiciones de Michoacán y conservar esta cultura [...] en más de 30 años empecé a trabajar con los niños [...] al tiempo organicé talleres sobre la ribera y me llevó a meter proyectos para pensar en dar a conocer el nombramiento de Pátzcuaro como pueblo mágico [...] así fue como me inicié y me vinculé al Consejo Ciudadano [...] (BLANCAS, 2018)."
\end{abstract}

En este proceso, además, enseñó a la juventud a trabajar el barro y crear artesanías. Al jubilarse, se dedicó, con más fervor, a la conservación de la cultura organizando recorridos culturales para los maestros y su alumnado de las escuelas de Pátzcuaro y de las comunidades aledañas. Desde entonces coleccionó sistemáticamente información y fotografías sobre los monumentos coloniales de la región y que posteriormente se publicaron en libros.

La biblioteca Gertrudis Bocanegra se encuentra en lo que fue una iglesia de un convento franciscano del Siglo XVl, y por órdenes del entonces presidente Lázaro Cárdenas en 1934 fue transformado en biblioteca. Su funcionamiento ha estado a cargo de la Dirección General de Bibliotecas de la SEP y administrado en sus servicios básicos por el Ayuntamiento de 
Pátzcuaro. El mural fue pintado en 1943 por Juan $O$ 'Gorman y, desde entonces, es considerado un monumento histórico y patrimonio nacional propiedad del Instituto Nacional de Antropología e Historia (INAH).

Como hemos aludido, en el momento que se fundó el Consejo la bibliotecaria explicó que no sólo su labor se limitó a atender las labores bibliotecarias, sino que también llevó a cabo acciones para generar acción colectiva e incidencia hacia las autoridades y la sociedad civil con la finalidad de llevar a cabo un proyecto integral de restauración. De inicio, se inició con el rescate de toda la información sobre el edificio histórico y se elaboró un informe de las intervenciones realizadas desde el año de 1985, para dar a conocer el problema a las autoridades e instancias responsables.

En este proceso, Gloria Blancas López expresó que debido a que el inmueble sufría de goteras durante la época de lluvia, por el mal estado de las tejas y el daño de la bóveda de madera y se empezó a movilizar el tema en las instancias del municipio y del INAH. Con base en los trabajos y fotografías sobre el deterioro del edificio se buscaron aliados para lograr una intervención de raíz y salvar a la brevedad el edificio. Por ejemplo, se buscó financiamiento con el grupo de empresarios hoteleros y los ciudadanos americanos jubilados que radican en Pátzcuaro, y que años atrás habían apoyado a la biblioteca con donaciones de libros.

Finalmente, en el año 2017, el Consejo logró realizar una reunión con el delegado del INAH en Michoacán y el presidente municipal de Pátzcuaro, con los que se acordó el apoyo inicial para los trabajos de reparación de techo, así como revisar con especialistas el deterioro del edificio para llevar a cabo un proyecto seguro de reparación. En esa ocasión, el diario Monitor Expreso de Michoacán destacó que:

"El presidente municipal, Víctor Manuel Báez Ceja, acompañado por la Secretaria de Cultura, Silvia Figueroa Zamudio, y el Delegado del Instituto Nacional de Antropología e Historia (INAH), Jacinto Robles Camacho, acordaron realizar los trabajos de restauración de tres edificios históricos, de los que destaca la biblioteca pública "Gertrudis Bocanegra [...] se explicó la problemática por la que atraviesa el edificio el cual por falta de mantenimiento ha sufrido deterioro en su estructura, pero acordaron realizar las gestiones para 
trabajar en la restauración a la brevedad ( $M E, 30$ de marzo de 2017)."

Como la propuesta del presidente municipal fue que el Ayuntamiento pone la mano de obra si el Consejo pone los materiales, los miembros del Consejo escribieron solicitudes de apoyo a diferentes instituciones financieras del país para buscar el financiamiento necesario. Inesperadamente, y aprovechando la visita del secretario de educación pública federal al Centro de Cooperación Regional para la Educación de Adultos en América Latina y el Caribe (CREFAL) para inaugurar la plaza cultural Kópikwa y la librería del Fondo de Cultura Económica, se logró invitar a este funcionario a un recorrido de la biblioteca y se le compartió la necesidad de contar con el apoyo institucional para salvar el edificio y el mural, y para transformar a la biblioteca con mejores recursos educativos y tecnológicos.

Gracias al interés del Secretario de Educación y su esposa, los miembros del Consejo lograron obtener los recursos necesarios, y se acordó que el CREFAL fuese la instancia para coordinar el proyecto de restauración y reparación, así como administrar los recursos económicos para rehabilitar el lugar y se expresó la necesidad de articular esfuerzos con las autoridades locales y federales, así como con la ciudadanía del este lugar. En la entrevista realizada a Gloria Blancas se pudo reconocer un interés importante para llevar a cabo el proyecto y también se expresó la necesidad de lograr que la sociedad civil conociera, en su momento, los trabajos y otros temas relacionados con la construcción de ciudadanía. Como bien lo expresa Blancas:

"[...] es importante que entre la sociedad civil se den a conocer los trabajos de la biblioteca. Nosotros tenemos proyectado invitar a la ciudadanía para que vea cómo va a quedar la biblioteca y que se sume al trabajo con las autoridades para que le den solución para el arreglo de la calle lateral del edificio. Pero necesitamos hablar, que nos escuchen y demostrar que la ciudadanía se interesa en esto [...] CREFAL ahora está haciendo una intervención de raíz [...] estamos al pendiente como Consejo Ciudadano (BLANCAS, 2018)."

De esta manera, de junio a septiembre de 2018, se inició con el trastejado y la limpieza de la cubierta de la biblioteca y se llevó a cabo la 
reconstrucción de la torre del edificio, así como se llevaron a cabo trabajos de carpintería para el cambio de madera dañada y se restituyeron los baños del lugar. También se llevó a cabo una limpieza profunda de los libreros y de los libros y se reemplazó la pintura de las paredes, se restauró el jardín y se rehabilitó la luz eléctrica en las mesas de lectura; se adquirieron muebles nuevos para el personal (archiveros, sillones, escritorio) y se adquirió equipo de cómputo, entre otros materiales didácticos y principalmente acervo bibliográfico actualizado.

Aunque en la actualidad el proyecto de rehabilitación se encuentra en marcha, es importante subrayar que estas acciones realizadas por el CREFAL y la SEP no hubieran sido posibles sin las actividades de organización e incidencia pública del Consejo para influir en la toma de decisiones y motivar entre la ciudadanía del lugar su interés en las labores relacionadas con la preservación de la cultura y educación. En este camino, el Consejo, poco a poco, se ha venido constituyendo como un motor para impulsar la participación solidaria y motivar la construcción de ciudadanía desde una perspectiva no sólo de cooperación e intercambio con organizaciones sociales, públicas y privadas, sino fundamentalmente para generar conciencia sobre la importancia que tiene la intervención de la sociedad civil en las políticas públicas y en las acciones que van más allá de la colaboración oficial donde el tema de la preservación de la cultura y rescate de edificios coloniales constituyen el motivo que anima su acción colectiva desde una filosofía generosa y por el bien de la comunidad.

La rehabilitación ecológica del Río Guani. Don Vasco de Quiroga, visitador de Michoacán y primer obispo de la diócesis de Michoacán, trasladó la sede del obispado a Pátzcuaro por la abundancia de manantiales y por el pequeño río limpio y cristalino que traversa toda la comunidad de sur a norte hasta llegar al Lago de Pátzcuaro en 1537. Hacia el 2018, este lugar no sólo ha sufrido una transformación histórica, de rural a urbana radical, sino el remoto rio transparente hoy se caracteriza por ser un lugar donde confluyen aguas negras y residuos sólidos que emite la ciudad, y 
donde se pueden encontrar, sin exagerar, desde botellas de plástico, viviendas, llantas, animales y, en algunas ocasiones, personas muertas.

En un primer intento por visibilizar entre la sociedad civil el tema de la contaminación del rio Guani, algunos miembros del Consejo durante la década de los 80 , ya habían advertido la necesidad de rescatar al rio y transformarlo en un espacio atractivo de la ciudad. No obstante, fue hasta el año de 2016, cuando los miembros del Consejo comenzaron a recopilar toda la información disponible sobre el rio en los archivos del Municipio de Pátzcuaro y en el organismo operador de agua potable y en el Instituto Mexicano de Tecnología del Agua. En este proceso, se empezó a generar acción colectiva para solicitar a las autoridades acciones concretas para detener el deterioro y colaborar en el diseño de un proyecto para evitar la contaminación hacia la cuenca del lago de Pátzcuaro.

Aunque los miembros del Consejo se esforzaron por tener toda la información disponible del problema y se realizó un presupuesto y cronograma de trabajo, desafortunadamente no se llevó a cabo debido que existió un escaso apoyo del municipio. Además, entre sus miembros se advirtió una cierta tendencia hacia la "politización del problema del rescate del Lago de Pátzcuaro." A este respecto, Vargas y Huerto (2014: 186) ya habían señalado en el año de 2014, que entre pescadores y líderes comunitarios se expresaba que las intervenciones en el lago de Pátzcuaro en general $y$ en el sector pesquero en particular, en realidad persiguen fines políticos más que ambientales. Asimismo, y con entrevistas realizadas en la zona, subrayaron que el dinero que reciben funcionarios, organizaciones no gubernamentales y líderes políticos con programas han demostrado poca efectividad. Así, y ante la posibilidad de caer en un círculo vicioso ante el uso político del tema, la posición del Consejo ha sido -según su presidente René Ortiz- mantenerse al margen y buscar nuevos caminos, ya que sus estatutos dicen que ni la religión, ni la política deben influir en los proyectos.

Hacia el año de 2017, el Consejo llevó a cabo otras estrategias de movilización para incidir nuevamente en la sociedad civil por medio del concurso público intitulado "Salvemos al Rio Guani". Este proyecto se 
caracterizó por promover la participación en las escuelas de nivel secundaria y media superior de Pátzcuaro y sus alrededores. El proyecto planteó cuatro categorías de intervención: fotografías, ensayos, cuentos o poesías, dibujos o pinturas. La finalidad principal fue realizar un ejercicio de prospectiva para imaginar un río limpio, así como crear alternativas y tener un ejercicio de imaginación para conservar el rio y generar un ecosistema con vegetación abundante y atractivo para la comunidad. Algunas notas aparecidas en el Diario Cambio de Michoacán destacaron lo siguiente:

\begin{abstract}
"Con el fin de rescatar y embellecer el Río Guani, el cual en el pasado fue un afluente de aguas cristalinas y en la actualidad está convertido en captación de aguas negras y residuales de gran parte de drenajes domiciliarios y públicos, el Consejo Ciudadano de la Región Lacustre ha lanzado una convocatoria dirigida principalmente al sector educativo con el fin de que presenten propuestas, proyectos y alternativas para regresar a sus orígenes este arroyo de aguas negras y convertirlas nuevamente en aguas limpias. En caso de lograr su rescate y limpieza a lo largo de los aproximadamente siete kilómetros que tiene de longitud y que desemboca, ya sea en las plantas tratadoras ubicadas a la orilla del Lago de Pátzcuaro o bien directamente al manto acuático, en un futuro no lejano se pudiera caminar por sus costados de este afluente rodeado de naturaleza y a la vez hacerlo patrimonio natural de Pátzcuaro (MARTíNEZ, 2017)."
\end{abstract}

El Consejo, generosamente, realizó y distribuyó carteles en todas las escuelas de la región para visibilizar el tema, pero la respuesta fue muy baja debido a que sólo se registró la participación de 15 estudiantes, la mayoría de la comunidad de Huecorio, donde los maestros ya trabajaban el tema. En esta causa, y para la entrega de premios, el Consejo obtuvo el apoyo del CREFAL y de la directora de la biblioteca pública Gertrudis Bocanegra y se logró presentar las obras del alumnado durante una semana en la biblioteca donde se entregaron también los premios.

Debido a la escasa participación ciudadana del proyecto anterior, en marzo de 2018, el Consejo llevo a cabo la realización del "Foro Regional de Rehabilitación Ecológica de Ríos y Lagos de México." El propósito del evento fue "generar un espacio de análisis y reflexión en torno a la contaminación y rehabilitación de ríos y lagos de México a la luz de experiencias y propuestas de organizaciones de la región central de país (CCRLP, 2018)." Asimismo, la 
propuesta consideró la necesidad de compartir el problema hacia una comunidad especializada e impulsar -desde otro ángulo- la colaboración entre escuelas, organizaciones civiles e instituciones de carácter técnico y científico donde se logró la colaboración del CECYTEM de Huecorio, la Preparatoria Felipe Carrillo Puerto, el Tecnológico Superior de Pátzcuaro y del CREFAL para llevar a cabo el evento.

Este proyecto es relevante, sobre todo, porque se logró una asistencia significativa de científicos, maestros y alumnos entablando un diálogo, que abarcó la explicación de la utilidad de la plataforma internacional para el manejo integral de las cuencas de ríos y lagos, el abuso del agua en la agricultura industrial, y la importancia del enfoque de gobernanza ambiental, entre otros temas. En este evento presentaron 20 científicos, 8 maestros y 12 alumnos los resultados de sus investigaciones y se logró que 25 equipos de estudiantes de escuelas técnicas expusieran sus ideas en carteles. Un total de 357 personas se registraron en el evento. El foro se complementó con una obra de teatro referida al rio Guani intitulada "Verte así me duele: Propuesta teatral de sensibilización ciudadana sobre la contaminación del agua", la cual fue elaborada por un grupo de teatro "Los Achoques" del CECYTE de Huecorio, bajo la dirección de un voluntario francés con doctorado en estudios teatrales por la Universidad de Paris. Al final del evento, los fundadores de la Red Mexicana de Cuencas Hidrográficas (REMEXCU) redactaron una "Declaratoria con un manifiesto de ocho afirmaciones con relación al cuidado de las cuencas de aguas continentales de México", seis pronunciamientos en relación con los ríos y lagos de México, así como siete pronunciamientos a favor del río Guani y el lago de Pátzcuaro (http://remexcu.org/)."

Con base en lo anterior, se puede deducir que los esfuerzos para lograr la rehabilitación del Rio Guani durante los tres primeros años del Consejo no obtuvieron un impacto favorable al problema esencial, pero, por otro lado, se logró insertar el tema y problema público específico en la agenda de gobierno local y federal, así como motivar la intervención de múltiples 
actores en la elaboración de soluciones y acciones para atender un problema público.

Es importante mencionar que el proyecto en la actualidad sigue en curso y existe un cierto interés de continuar la colaboración en centros escolares de la región, así como mantener el apoyo de miembros de la REMEXCU para generar proyectos con otras instituciones nacionales e internacionales y diseñar un proyecto integral de rescate del rio Guani. Asimismo, se está desarrollando un estudio de opinión pública entre la población que habita en los alrededores del rio para conocer su sentir y percepción, así como elaborar junto con los vecinos propuestas pertinentes hacia la agenda de gobierno. El objetivo es transformar el escenario actual por medio de la participación solidaria y construir confianza entre la comunidad para que se pongan en práctica los derechos sociales y políticos que tienen los ciudadanos para lograr un propósito común.

\section{CONSIDERACIONES FINALES}

El análisis sobre la relación entre actores sociales e institucionales en el contexto de las perspectivas teórico-analíticas del gobierno abierto en México, y especialmente en el Estado de Michoacán, advierte la necesidad de revisar y discutir con mayor profundidad qué se entiende por la noción de gobernanza intercultural y cuál es su vinculación con los procesos de construcción de ciudadanía y el establecimiento de derechos sociales en México. En este contexto la gobernanza intercultural propone, de alguna manera, el fortalecimiento de las capacidades institucionales para analizar la diversidad cultural y proponer mejores diseños político-institucionales a la gestión de gobierno, así como lograr una mejor distribución de recursos entre los grupos y organizaciones de la sociedad civil. La gobernanza intercultural aporta innovación hacia la gestión pública al considerar una perspectiva más abierta a los temas, preocupaciones y casos de éxito en que la participación política de los ciudadanos produce un impacto en el desarrollo de la comunidad. 
En este trabajo se intentó dar un enfoque propositivo y significativo al estudio de las organizaciones de la sociedad civil que nacen con base en la reflexión social y la participación solidaria, así como sistematizar una parte de la experiencia del Consejo Ciudadano de la Región Lacustre de Pátzcuaro ubicado en el Estado de Michoacán, México, en los procesos de incidencia pública donde lo consideramos como un motor para la construcción de ciudadanía y eje fundamental para la acción colectiva entre las organizaciones de la sociedad civil a nivel municipal y local.

En este sentido, y pese a que los trabajos del Consejo siguen en curso, es necesario pensar que lo más importante de una asociación civil que quiere trabajar por el bien de la comunidad sin fines de lucro es generar voluntad, mantener la constancia y tener paciencia ante las innumerables horas de trabajo que se invierten y que serán únicamente remuneradas con los pequeños y grandes logros que se obtengan del trabajo colectivo.

El proyecto de la Biblioteca Pública Gertrudis Bocanegra tiene muchos aliados, pero es importante subrayar la necesidad de una mayor cooperación entre actores sociales, públicos y privados para lograr una perspectiva integral y valorar los esfuerzos del proyecto hacia un público más amplio e interesado por el lugar. En este marco, el trabajo del Consejo no sólo es promover iniciativas que tengan como finalidad la mejora del espacio público, sino coadyuvar en procesos de cambio educativo y construcción de ciudadanía desde un enfoque de filantropía, voluntariado y participación solidaria en beneficio de la comunidad.

En el caso de la rehabilitación de la cuenca del Río Guani se puede concluir que, hasta este momento, es imposible contar las horas de trabajo de todos los involucrados que con mucho esmero han donado su tiempo en tareas para sensibilizar y crear conciencia entre la ciudadanía sobre la importancia de preservar el río como un recurso natural importante y que le puede dar otra cara al llamado Pueblo Mágico de Pátzcuaro. En este caso, es muy importante no sólo visibilizar el tema en la agenda pública y de gobierno, sino desarrollar estrategias de movilización más eficientes para intentar incidir en actores con poder y decisión pública y que cuentan con 
los recursos económicos para llevar a cabo el proyecto de forma congruente y efectiva.

En los últimos tres años, y hacia el 2019, la labor del Consejo es relevante, pero es necesario fortalecer el trabajo de la asociación a nivel de su dirección y estructura interna, así como definir un plan estratégico más claro con líneas de acción que permitan estimar el alcance de los proyectos y su articulación con diversas instituciones y organizaciones comunes. No obstante, y con las evidencias realizadas, es posible advertir que el balance es favorable en la medida de que se ha logrado entrever el problema de la cuenca del Río Guani en la comunidad y se construyeron vínculos para desarrollar otros proyectos a largo plazo. Una relación importante que se logró fue la establecida con la REMEXCU donde el Consejo tiene acceso a la consulta con expertos que trabajan la rehabilitación de cuencas y conocen muy bien el tema para lograr la implementación del proyecto y cumplir el imaginario de rescate del río.

Asimismo, se ha logrado considerar en la agenda de trabajo del CREFAL la posibilidad de obtener financiamiento y apoyar la realización de otros eventos vinculados al diseño de políticas pública locales y regionales. Por otro lado, el Consejo presentó, en agosto de 2018, los avances de los trabajos realizados a la Fundación Gonzalo Río Arronte (http://www.fgra.org.mx/) con la intención de interesar a la Fundación de apoyar los proyectos con recursos económicos en beneficio de la rehabilitación ecológica del lugar.

En suma, es posible aseverar que, hasta este momento, el Consejo constituye una oportunidad de asociación para la acción colectiva que busca no sólo coadyuvar al impulso de la participación solidaria, sino que se propone, de alguna manera, construir ciudadanía efectiva. Si bien este proceso es aún limitado, es posible concluir que entre sus miembros existe una perspectiva de bien común, filantrópica y ciertamente voluntaria para motivar el cambio social.

Hacia el futuro se requiere de un fuerte trabajo de concientización y armonización política entre la comunidad patzcuarense y público interesado 
en los proyectos, lo cual implica la suma de mayores esfuerzos e integrar diferentes visiones, objetivos, responsabilidades y competencias institucionales para hacer realidad los enfoques y enfrentar los retos que plantea el desarrollo local, el combate a la pobreza e impulsar la calidad de la participación solidaria en esa región de México.

Por último, no está de más señalar que en comunidades como Pátzcuaro, el Estado de Michoacán, México, la gobernanza intercultural adquiere uma connotación especial debido a la pluralidad social existente en la región purépecha, así como a las movilizaciones sociales registradas por grupos y organizaciones civiles que trabajan en la construcción de políticas públicas desde una perspectiva autónoma, integral y solidaria en beneficio de la población más vulnerable.

\section{BIBLIOGRAFÍA}

AGUILAR VILLANUEVA, L. F., Gobernanza y gestión pública, México, Fondo de Cultura Económica, 2006.

ÁLVAREZ, L. Institucionalidad y autonomía de la participación ciudadana en Ciudad de México. Alcances y desafíos, en Controversia No. 191, diciembre 2008, Bogotá, Colombia, 2008.

ANDER-EGG, E. Repensando la investigación-acción participativa, Grupo Lumen Humanitas, México, 2003.

BUTCHER, J.; VERDUZCO, G. Acción voluntaria y voluntariado en México, México, Fundación Telefónica, 2016.

CABRERO, E. Acción pública y desarrollo local, México, Fondo de Cultura Económica, 2005.

CANTO CHAC, M. Participación ciudadana en las políticas públicas, México, Siglo XXI Editores, 2010.

COBB, R.; ROSS, J.; ROSS, M. H. Agenda Building as a Comparative Political Process,

The American Political Science Review, Vol. 70, No. 1. (March), pp. 126-138, 1976.

CONTRERAS, L. La gestión ambiental en los municipios de Pátzcuaro y Quiroga, Michoacán, México, Tesis de Maestro en Administración Integral del Ambiente, El Colegio de la Frontera Norte, 2008.

CUNILL, N. Participación ciudadana, dilemas y perspectivas para la democratización de los Estados latinoamericanos, Caracas, CLAD, Centro Latinoamericano de Administración para el Desarrollo, 1991.

DIARIO MONITOR, Acuerdan trabajar en el rescate de edificios históricos del municipio de Pátzcuaro, Monitor Expreso de Michoacán, marzo, 2017. Disponible en https://www.monitorexpresso.com/acuerdan-trabajar-rescate-edificios-historicosdel-municipio-patzcuaro/

GONZÁLEZ BOMBAL, I.; VILLAR, R. Incidencia de la sociedad civil en políticas públicas, Mimeografiado, Universidad de San Andrés, 2005. 
GONZÁLEZ IBARRA, M. R. Participación política y agenda de gobierno: un análisis a partir de casos de organizaciones sociales en México y la Ciudad de México, México, Instituto de Administración Pública del Estado de México, 2011.

HUERTO, R.; VARGAS, S. Estudio ecosistémico del Lago de Pátzcuaro: aportes en gestión ambiental para el fomento del desarrollo sustentable. México, Instituto Mexicano de Tecnología de Agua, 2014. Disponible en:

http://repositorio.imta.mx/handle/20.500.12013/1701 ?locale-attribute=en

KNIGTH, B. El valor de la filantropía comunitaria. Resultados de una consulta,

Fundación, Aga Khan Foundation USA, 2012. Disponible en

https://www.mott.org/wp-

content/uploads/2014/01/thevalueofcommunityphilanthropy_SPN.pdf

MARTíNEZ, A., Convocan a salvar el rio Guani, en Diario Cambio de Michoacán,

mayo, 2017. Disponible en http://www.cambiodemichoacan.com.mx/nota-n23698

MILLER, $V$. Desafíos y lecciones de la incidencia política. Interrogantes e Implicaciones para Programas de Acción, Capacitación y Apoyo Financiero.

Documento de trabajo, 2013; consultado el 15 de agosto de 2016. Disponible en https://adicnicaragua.files.wordpress.com

NATERA, A. Nuevas estructuras y redes de gobernanza, Revista Mexicana de Sociología, Vol. 67, No. 4, octubre-diciembre, 2005, pp. 755-791.

PÁGINA COMUNITARIA PURÉPECHA (s/f), Recuperado el 3-08-2018.

http://www.purepecha.mx/threads/6169-Poes\%C3\%ADa-P-urh\%C3\%A9-Uanancha-

P-urh\%C3\%A9pecha-Princesa-Pur\%C3\%A9pecha

PROGRAMA DE LAS NACIONES UNIDAS PARA EL DESARROLLO (PNUD), Ciudadanía política: Voz y participación ciudadana en América Latina, Buenos Aires: Siglo Veintiuno Editores, 2014.

REY GARCÍA, M. Filantropía y participación cívica en el albor del siglo XXI, en Participación Cívica y Filantropía No. 872, 2013.

REY-GARCÍA, M. Filantropía y participación en el albor del siglo XXI, Revista de Economía 972, Recuperado el 10 de agosto de 2018. Disponible en:

https://dialnet.unirioja.es/servlet/articulo? codigo $=4609601$

SAHUÍ, A. (coordinador), Gobernanza y sociedad civil. Retos democráticos. México, Ediciones Coyoacán, 2009.

VARGAS-HERNÁNDEZ, J. G. Gobernanza Intercultural, Revista Doxa, Vol. 6, No. 11, Universidad Autónoma de Chihuahua, México, 2016.

\section{ENTREVISTAS REALIZADAS}

BLANCAS LÓPEZ, G. (2018), miembro fundador y secretaria del Consejo Ciudadano de la Región Lacustre de Pátzcuaro, realizada en la Ciudad de Pátzcuaro, Estado de Michoacán, el 10 de agosto.

ORTIZ ROSILLO, R. (2018), miembro fundador y presidente del Consejo Ciudadano de la Región Lacustre de Pátzcuaro, realizada en la Ciudad de Pátzcuaro, Estado de Michoacán, el 10 de agosto.

ILSE BRUNNER SCHOENEMANN (2018), miembro del Consejo Ciudadano de la Región Lacustre de Pátzcuaro, realizada en la Ciudad de Pátzcuaro, y activista a favor del medio ambiente y el desarrollo comunitario en el Centro de Cooperación Regional para la Educación de Adultos en América Latina y el Caribe (CREFAL).

Recebido em: 06 fevereiro de 2019 Aprovado em: 25 de agosto de 2019 